\title{
Physically Unclonable Surfaces via Dewetting of Polymer Thin Films
}

\author{
Neslihan Torun, Ilker Torun, Menekse Sakir, Mustafa Kalay, and M. Serdar Onses* \\ Cite This: ACS Appl. Mater. Interfaces 2021, 13, 11247-11259 \\ Read Online
}

ABSTRACT: From anti-counterfeiting to biotechnology applications, there is a strong demand for encoded surfaces with multiple security layers that are prepared by stochastic processes and are adaptable to deterministic fabrication approaches. Here, we present dewetting instabilities in nanoscopic (thickness $<100 \mathrm{~nm}$ ) polymer films as a form of physically unclonable function (PUF). The inherent randomness involved in the dewetting process presents a highly suitable platform for fabricating unclonable surfaces. The thermal annealinginduced dewetting of poly(2-vinyl pyridine) (P2VP) on polystyrenegrafted substrates enables fabrication of randomly positioned functional features that are separated at a microscopic length scale, a requirement set by optical authentication systems. At a first level, PUFs can be simply and readily verified via reflection of visible light. Area-specific

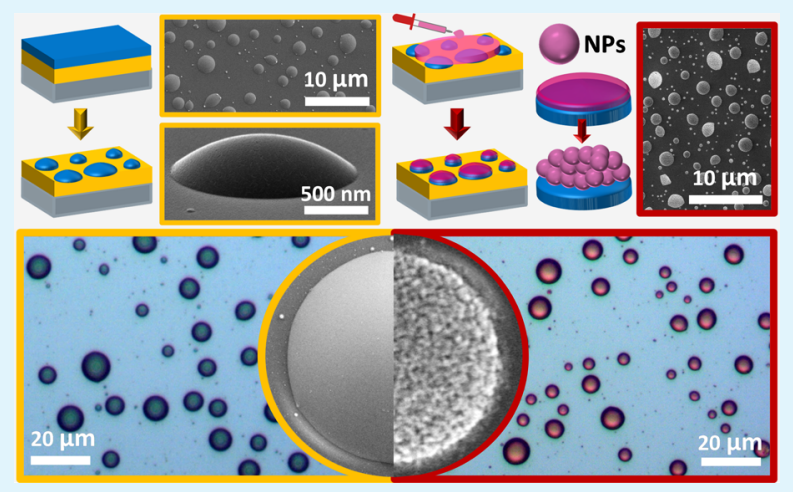
electrostatic interactions between P2VP and citrate-stabilized gold nanoparticles allow for fabrication of plasmonic PUFs. The strong surface-enhanced Raman scattering by plasmonic nanoparticles together with incorporation of taggants facilitates a molecular vibration-based security layer. The patterning of P2VP films presents opportunities for fabricating hybrid security labels, which can be resolved through both stochastic and deterministic pathways. The adaptability to a broad range of nanoscale materials, simplicity, versatility, compatibility with conventional fabrication approaches, and high levels of stability offer key opportunities in encoding applications.

KEYWORDS: physically unclonable functions, dewetting, polymer films, nanoparticles, plasmonics, SERS

\section{INTRODUCTION}

The concept of physically unclonable functions (PUFs) refers to the exploitation of a physical system in authentication applications for generating a response as a result of an input challenge. ${ }^{1,2}$ The physical system in the PUF has a unique and unclonable response to a particular challenge. PUFs can be constructed using intrinsically stochastic processes, which produce random features. ${ }^{3}$ The strong interest on these systems emerges from the impossibility of predicting the response by the manufacturer and third parties. ${ }^{4}$ There are two strengths of this approach for producing security labels in comparison with the deterministic ${ }^{5,6}$ fabrication of encoded surfaces based on geometrically defined patterning (i.e., barcodes) of taggant materials. First, the intrinsic randomness of PUFs makes the reproduction of the labels impossible, whereas the deterministic labels can, in principle, be reproduced by the adversaries. Second, PUFs are particularly suitable for applications, where there is a need to define a unique label for each object rather than attaching the same label to a large group of objects. Physically unclonable security labels are critically important for information security and anticounterfeiting applications. The former application is critically important for our modern era that relies on information technology. The latter relates to the counterfeiting, which is one of the largest illicit economies with a dramatic negative impact on the economy, health, and security of our society. The unique characteristics of nanomaterials, in particular, are highly suitable for fabrication of PUF-based security layers. Different strategies including laser scattering patterns, ${ }^{3}$ random deposition of colloidal nanomaterials ${ }^{7,8}$ and fluorescent proteins, ${ }^{9}$ stochastic pattern formation within ink-jet-printed quantum dot features, ${ }^{10}$ spontaneous folding of plasmonic gels, ${ }^{11}$ lanthanide ion-doped zeolites dispersed in a polymer film, ${ }^{12}$ self-assembly of carbon nanotubes, ${ }^{13}$ chemical vapor deposition of two-dimensional materials, ${ }^{14}$ variations in the switching of memristor devices, ${ }^{15}$ and wrinkling-based synthetic fingerprints ${ }^{16,17}$ have been reported as PUF-based labels. An ideal approach should be universally applicable to different types of taggant nanomaterials and enable simple fabrication of robust physically unclonable security labels with the capability of integration with the deterministic patterning processes.

Received: September 18, 2020

Accepted: February 3, 2021

Published: February 15, 2021 

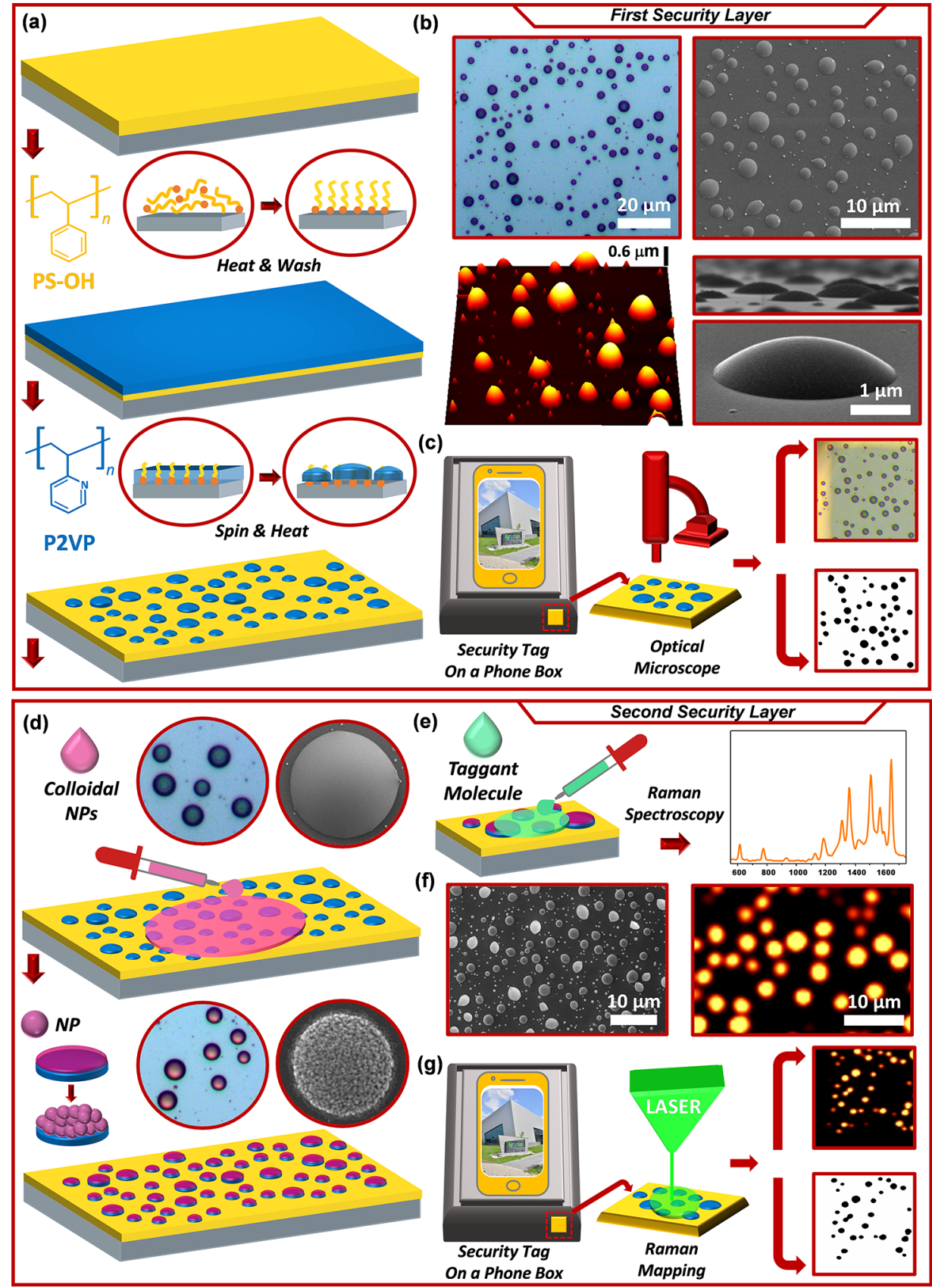

Figure 1. Dewetting instabilities in polymer thin films as a form of PUF. (a) Schematic illustration of the key steps of fabrication. The process starts with a grafted layer of PS, to provide appropriate surface energy to induce dewetting. The grafting is achieved by spin-coating followed by heating. A film of P2VP is then spin-coated and annealed (e.g., $250{ }^{\circ} \mathrm{C}$ for $5 \mathrm{~min}$ ) to generate randomly positioned P2VP features. (b) Optical microscopy (top left), SEM (top right), AFM (bottom left), and cross-sectional SEM (bottom right) images of dewetted P2VP features. (c) Image analysis from a product is depicted. (d) Assembly of colloidal gold NPs over the PUFs. Insets provide optical microscopy and SEM images before and after selective adsorption of NPs. (e) Schematic illustration for the deposition of probe molecules and Raman mapping of the surface. (f) SEM and Raman mapping images of the surface following deposition of gold NPs and probe molecules. Raman mapping shows the intensity at a position of $1649 \mathrm{~cm}^{-1}$. (g) Analysis of Raman mapping images is depicted.

Dewetting of polymers in thin $(<100 \mathrm{~nm})$ films is a wellknown instability that is commonly undesirable. Such films can easily lose their stability and rupture into separate structures when they have sufficient mobility gained through heating above the glass transition temperature of the polymer or swelling of the polymer with a solvent. ${ }^{18-20}$ For the majority of applications including functional coatings, ${ }^{21}$ nanolithography, ${ }^{22}$ sensors, ${ }^{23}$ and devices, ${ }^{24}$ there is a need for homogenous deposition of materials, and dewetting is an undesired phenomenon. The breakup of a continuous film into discrete features at micrometer and nanometer length scales, on the other hand, provides unique opportunities to organize materials on surfaces for different applications. ${ }^{25}$ The efforts to date mostly focused on directing the processes of dewetting to generate ordered patterns from the intrinsically random structures. ${ }^{26}$ Chemical and topographical templates and flow and electric fields have been utilized to generate ordered arrays of functional materials. ${ }^{27-30}$ To the best of our knowledge, the 
intrinsic randomness of the dewetted patterns has not been utilized as a form of PUF.

Herein, we propose that the dewetting instabilities in polymer thin films provide unprecedented opportunities in extremely simple yet highly effective fabrication of robust and unclonable security labels. The stochastic nature of the dewetting process that leads to random features is the basis of the approach presented in this study. A key characteristic is the proper length scale allowing verification of the random features by different types of microscopes that rely on optical phenomena. The dewetting of polymer films leads to droplets of varying sizes that are separated by several micrometers. According to the Rayleigh criteria, ${ }^{31}$ the minimum distance that can be resolved with an optical microscope is $\sim 200 \mathrm{~nm}$ and the distinction becomes practically challenging for separation distances less than $1 \mu \mathrm{m}$. Therefore, dewetted features can be verified by optical authentication systems that rely on reflection, scattering, fluorescence, and absorbance of light. In the following, we demonstrate dewetting of poly(2vinylpyiridine) (P2VP) on top of polystyrene (PS)-grafted substrates. The spontaneous dewetting occurs on the order of couple of minutes by thermal annealing at temperatures that range from 150 to $250{ }^{\circ} \mathrm{C}$. This process leads to random features with sizes and spacings tunable with the thickness of the P2VP films and annealing conditions. The first security layer can be simply verified using an optical microscope. To further complicate the encoding, nanomaterials can be selectively attached to the functional P2VP features through electrostatic interactions. We employ citrate-stabilized gold nanoparticles to generate plasmonically active sites for a surface-enhanced Raman scattering (SERS)-based encoding layer. In SERS, ${ }^{32,33}$ the plasmonic nanoparticles provide electromagnetic enhancement of the weak Raman scattering from taggant molecules. SERS has recently gained attention in authentication applications with its multiple modalities including molecular specific fingerprint vibrations, intensity variations, and polarization-dependent scattering. ${ }^{34-41}$ Following deposition of plasmonic nanoparticles and taggants, the random features can be observed by mapping the intensity of characteristic fingerprint vibrations of the molecules using Raman microscopy. Overall, dewetting instabilities in polymer thin films offer several unique advantages in fabrication of unclonable surfaces. (i) The fabrication is simply performed by spin-coating of a polymer film followed by thermal annealing. (ii) The approach can be readily applied to different nanomaterials, which have unique fluorescence, light scattering, and other properties. This universality stems from decoupling the patterning process from colloidal functionalization. P2VP, for example, can bind a broad range of nanoparticles with a net negative surface charge. (iii) The presented approach is compatible with massively parallel and conventional manufacturing (e.g., lithography) routes, suggesting their adaptation in device applications and hybrid security labels based on both stochastic and deterministic authentication pathways. (iv) The robust binding of nanomaterials to the randomly positioned features with strong chemical contrast enables fabrication of PUFs with a high signal-to-noise ratio, chemical stability, and low levels of contamination. (v) Dewetting instabilities offer a highly suitable platform for PUF applications through precise control over the composition, structure, size, and spacing of random features.

\section{RESULTS AND DISCUSSION}

Figure 1 presents the schematic illustration of the important steps and key results for fabrication of unclonable surfaces through dewetting of polymer films and assembly of colloidal nanoparticles. The first security layer is formed by dewetting of a thin film of P2VP over a PS-grafted substrate (Figure 1a). The spin-coating of a thin film of PS with a hydroxyl end functionality followed by thermal annealing and washing steps results in a PS-grafted substrate. The thermal annealing facilitates condensation reaction between the end terminus of the polymer and the silanol groups of the substrate, whereas the latter washing step removes the excess material. ${ }^{42}$ This process is important to obtain a relatively low-energy (41.1 $\mathrm{mN} / \mathrm{m}$, Figure S1) surface that is thermodynamically unfavorable for spreading of subsequent polymer layers. We then spin-coat a film of P2VP. The choice of P2VP stems from several considerations: (i) this polymer consists of a structural unit comprising a pyridine moiety, providing a universal attachment chemistry for colloidal nanomaterials of varying size, shape, geometry, and composition ${ }^{43,44}$ and (ii) P2VP has a surface energy $(54.5 \mathrm{mN} / \mathrm{m}$, Figure S1) higher than PS, making its thin films vulnerable to dewetting using moderate temperatures and sufficiently thick films. Heating these films at a temperature higher than the glass transition temperature of P2VP resulted in spontaneous dewetting of the films into randomly positioned features with sizes and separation distances that depend on the thickness of the P2VP film and annealing conditions. Figure $1 \mathrm{~b}$ presents optical, electron, and atomic force microscopy (AFM) images of P2VP films with a thickness of $25.5 \mathrm{~nm}$ dewetted on the PS-grafted substrate by heating at $250{ }^{\circ} \mathrm{C}$ for $5 \mathrm{~min}$. AFM and cross-sectional scanning electron microscopy (SEM) images reveal the non-planar and $3 \mathrm{D}$ nature of the P2VP features. The first security layer can be verified via optical microscopy. Figure 1c depicts the placement of such encoded surfaces on a box of an electronic device. The capture and binarization of an image are followed by authentication, as discussed in the following sections.

The complexity of the PUFs can be readily increased by incorporating colloidal NPs through the functional groups provided by P2VP (Figure 1d). We employ citrate-stabilized spherical gold NPs of varying sizes. The drop casting of a colloidal gold NP solution on the patterns is sufficient for selective adsorption of the particles over the P2VP features. Subsequent washing under sonication ensures removal of weakly bound particles that can be present on the PS regions. A remarkable characteristic of the fabricated labels is that the nanoparticles are firmly attached to the substrate that they can withstand washing in water under sonication. The pinkish color of P2VP features is a first indication of successful assembly of colloidal gold NPs. SEM imaging verifies highdensity adsorption of gold NPs over the randomly positioned patterns with almost complete absence over the background regions. Now, the PUFs are plasmonically active and can serve as sites for strong Raman scattering. A taggant molecule is deposited from an aqueous solution (Figure 1e). The choice of the taggant molecule presents an unlimited number of encoding possibilities that rely on fingerprint vibrations of molecules. The surface can now be scanned by Raman spectroscopy since colloidal gold NPs assembled on randomly positioned features selectively enhance the Raman scattering from the taggant molecule. A unique fingerprint Raman spectrum from the dewetted features should match the 
(a)
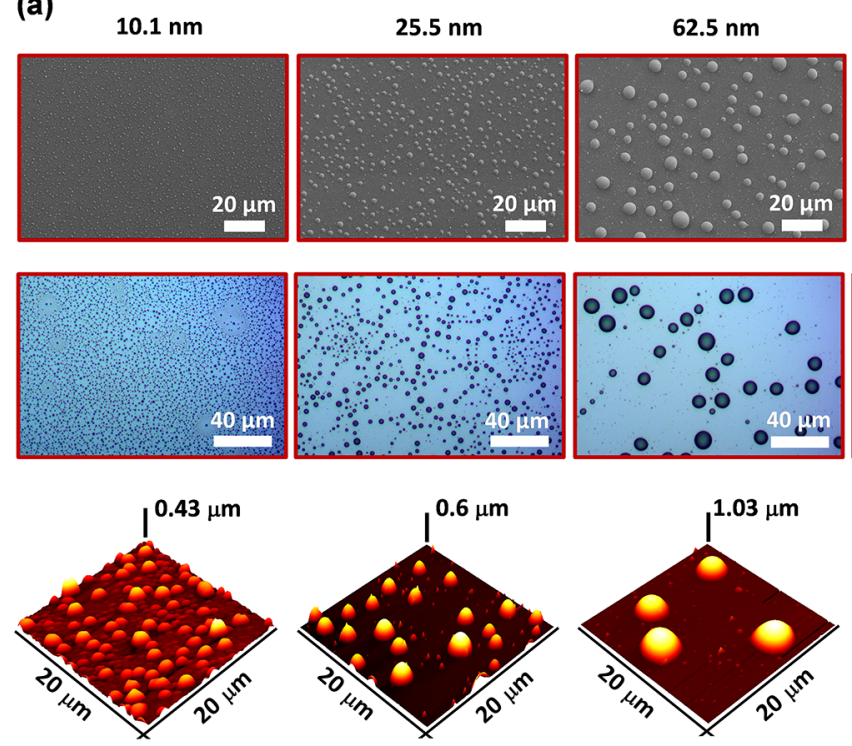

(c)

(b)

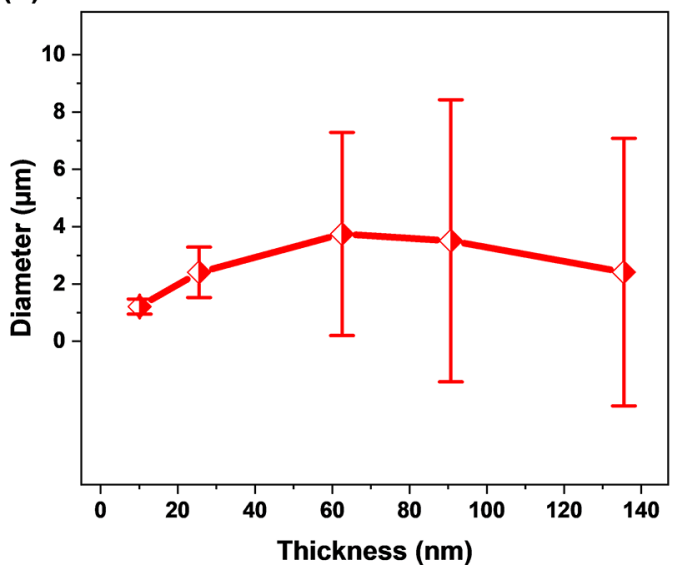

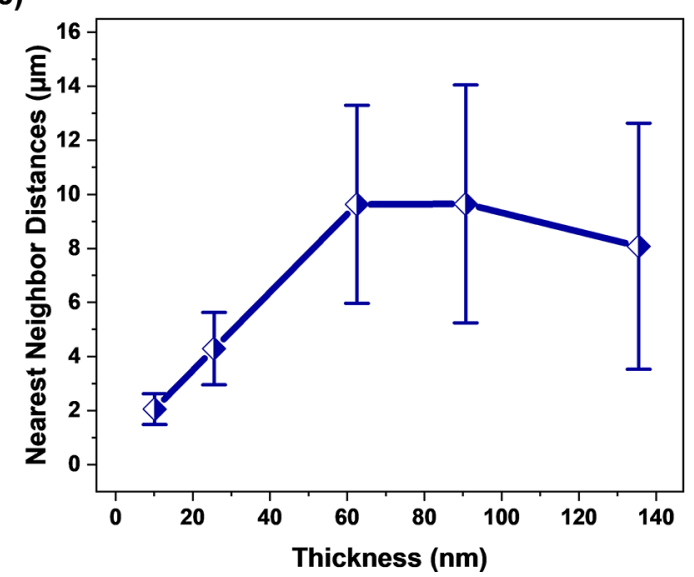

Figure 2. Effect of the thickness of the P2VP film on the size and separation distances of randomly positioned features. (a) SEM (top row), optical microscopy (second row), and AFM (third row) images of the surfaces prepared by spin-coating of the P2VP films followed by thermal annealing at $250{ }^{\circ} \mathrm{C}$ for $5 \mathrm{~min}$. The films were spin-coated on top of the end-grafted PS substrates from solutions of P2VP containing $0.5,1,2,3$, and 4 wt $\%$ polymer. The thickness of the P2VP film is given at the top of the images. (b) Diameter and (c) nearest neighbor distance of dewetted P2VP features as a function of the thickness of the P2VP film. The image analysis was performed in ImageJ and features with an area smaller than $1 \mu \mathrm{m}^{2}$ were not included in the analysis.

spectrum for the taggant molecule. In practical implementation of the presented encoding system, the rapid measurement of the Raman spectrum can be the primary verification of the chemical information.

Advanced verification of the encoded surface involves mapping the Raman scattering of the surface at a specific Raman shift peculiar to the taggant molecule (Figure 1f,g). The SERS activity exhibits a certain level of randomness due to varying lateral and out-of-plane dimensions of the features. The concentration of the taggant molecule can be intentionally varied to tune the Raman scattering intensity, presenting ways to further complicate the surface encoding. Therefore, a mapping image generated at the encoded Raman shift provides information in addition to spatial positions of the features that can be extracted through conventional light microscopy. The Raman mapping image verifies successful assembly of colloids and localized SERS response from the randomly positioned P2VP features. The position of bands that Raman mapping was taken together with the intensity of Raman signals can be programmed via selection of the probe molecule and its concentration, providing novel pathways for defining additional security layers. A similar representative image analysis from Raman mappings can verify the unclonable nature of the fabricated surfaces.

The thickness of the P2VP film is a highly critical parameter for controlling the diameter of dewetted features and their separation distances. An inherent advantage of the presented approach is that the size of features can be tuned over a broad range that spans dimensions from the scale of micrometers to nanometers. ${ }^{19}$ The stability of a polymer film on a solid substrate is dependent on the thickness of the film. ${ }^{45}$ Therefore, we first studied the effect of the thickness of the P2VP film on the PS-grafted substrate. The thickness of the film was controlled by varying the concentration of the solution for spin-coating. Figure 2 presents SEM, optical microscopy, and AFM images of the P2VP films of different thicknesses dewetted as a result of annealing at $250{ }^{\circ} \mathrm{C}$ for 5 min. All surfaces displayed water contact angles of $\sim 92^{\circ}$ 
(a)

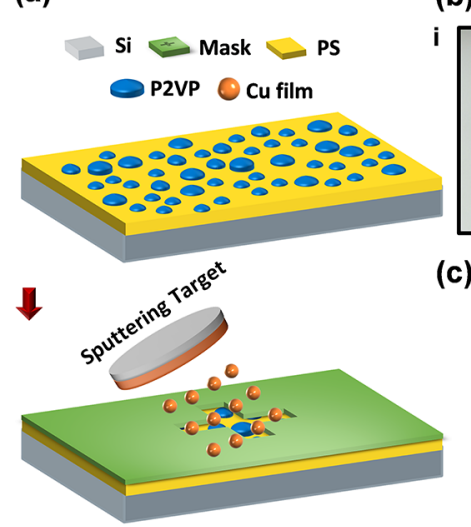

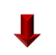

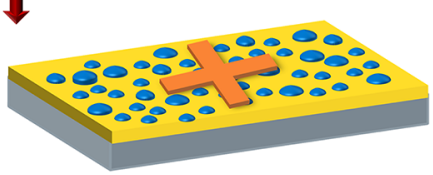

(d)

(e)

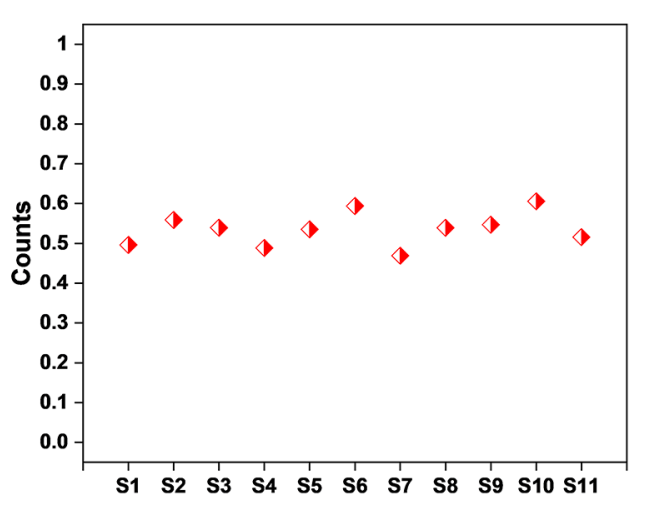

(b)
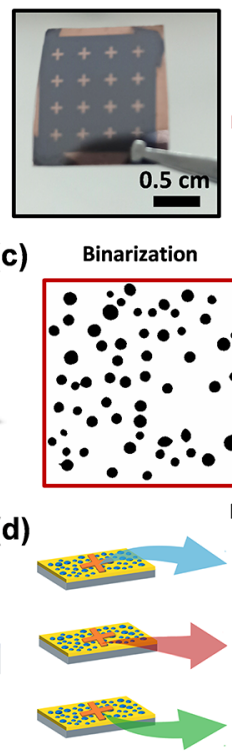
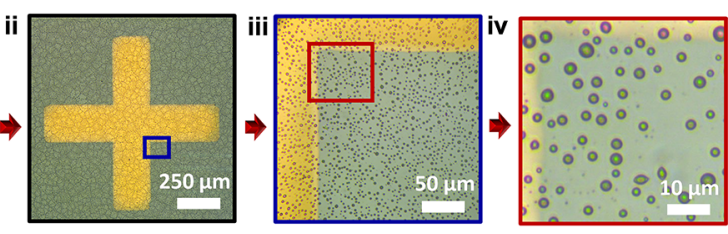

Key generation (256 bits)

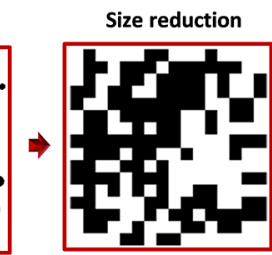

[10111100111011111001000100000011 10110010000101100010011000010110 01100000000110011001000001101001 10011010011011110000000001111000 10001001101110110011001011111111

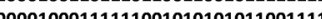
00001111001100011001000110100100000 10011011100100111111001001111111]

Key generation

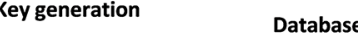

Authentication

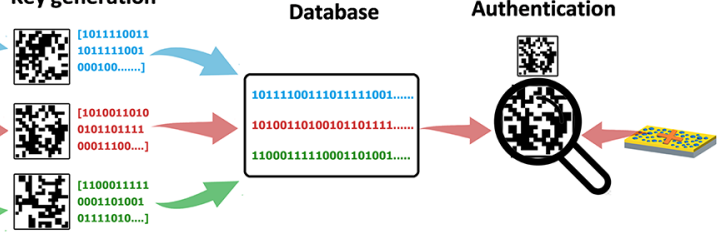

(f)

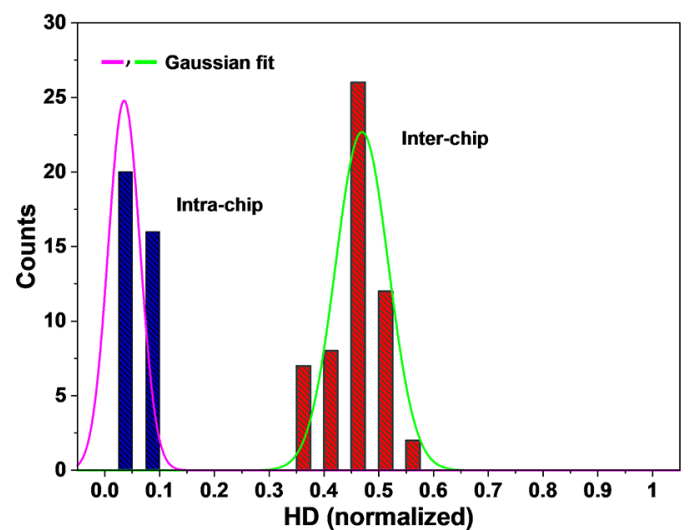

Figure 3. Authentication of the physically unclonable surfaces fabricated by dewetting of P2VP films followed by assembly of colloidal gold NPs. (a) Scheme illustrates definition of markers through physical vapor deposition of a copper film from a stencil mask. (b) Sequence of images taken from the sample. (i) Photograph of the silicon wafer with the PUFs and markers and (ii-iv) optical microscope images of the PUFs near the marker. (c) Binarization and size reduction of images for generation of a security key. (d) Schematic illustration of the authentication method. (e) Uniformity of bits obtained from 11 samples. (f) Distribution of Hamming distances. Inter-chip refers to the Hamming distance obtained among 11 samples. Intra-chip Hamming distance refers to the same sample challenged under five different conditions.

(Figure S2) upon dewetting. This wetting property is a result of the underlying end-grafted PS together with the roughness induced by the dewetted features. Both the diameter of the features and nearest neighbor distances become larger with the increasing thickness of the P2VP film. At a thickness of $\sim 10$ $\mathrm{nm}$, for example, the average size of dewetted features was 1.21 $\mu \mathrm{m}$ and the distance between nearest neighbors was $2.05 \mu \mathrm{m}$. Note that the size of features can be further reduced below 100 $\mathrm{nm}$ by varying the intrinsic properties of polymers and dewetting conditions. ${ }^{46}$ With the increase in the thickness, droplets as large as $12.6 \mu \mathrm{m}$ formed, along with the smaller features. The enlargement of the features is coupled with the increase in their out-of-plane dimension as confirmed via AFM. The analysis of images presented in Figure 2b,c shows the variation in the diameter of the features and their nearest neighbor distances as a function of the thickness of the P2VP film. Note that for thicker films, the arithmetic average of the diameter and nearest neighbor distance reaches a plateau and then exhibits a slight decrease. This behavior appears to be different from the images showing the presence of distinctly large dewetted features for the thicker films. This contrast arises from the low number of large dewetted features, resulting in an apparent decrease in the average diameter and nearest neighbor distance. The higher levels of standard deviation in the case of thicker films support this observation. The variation in the size of features is an advantage since this variation contributes to the randomness of the patterns. For practical applications of PUFs, the distances between the dewetted features should be large enough for their verification via optical microscopy and Raman mapping. Thermal annealing at a reduced temperature of $200{ }^{\circ} \mathrm{C}$ resulted in a similar behavior (Figure S3). Further reduction of the annealing temperature to $150{ }^{\circ} \mathrm{C}$ at a duration of $5 \mathrm{~min}$ resulted in incomplete dewetting as a result of the insufficient mobility of the polymer chains (Figure S4). Based on these results, $1 \% \mathrm{P} 2 \mathrm{VP}$ is used for spin-coating the films and the thermal annealing is performed at $250{ }^{\circ} \mathrm{C}$ for $5 \mathrm{~min}$ in the rest of the study. 
(a)

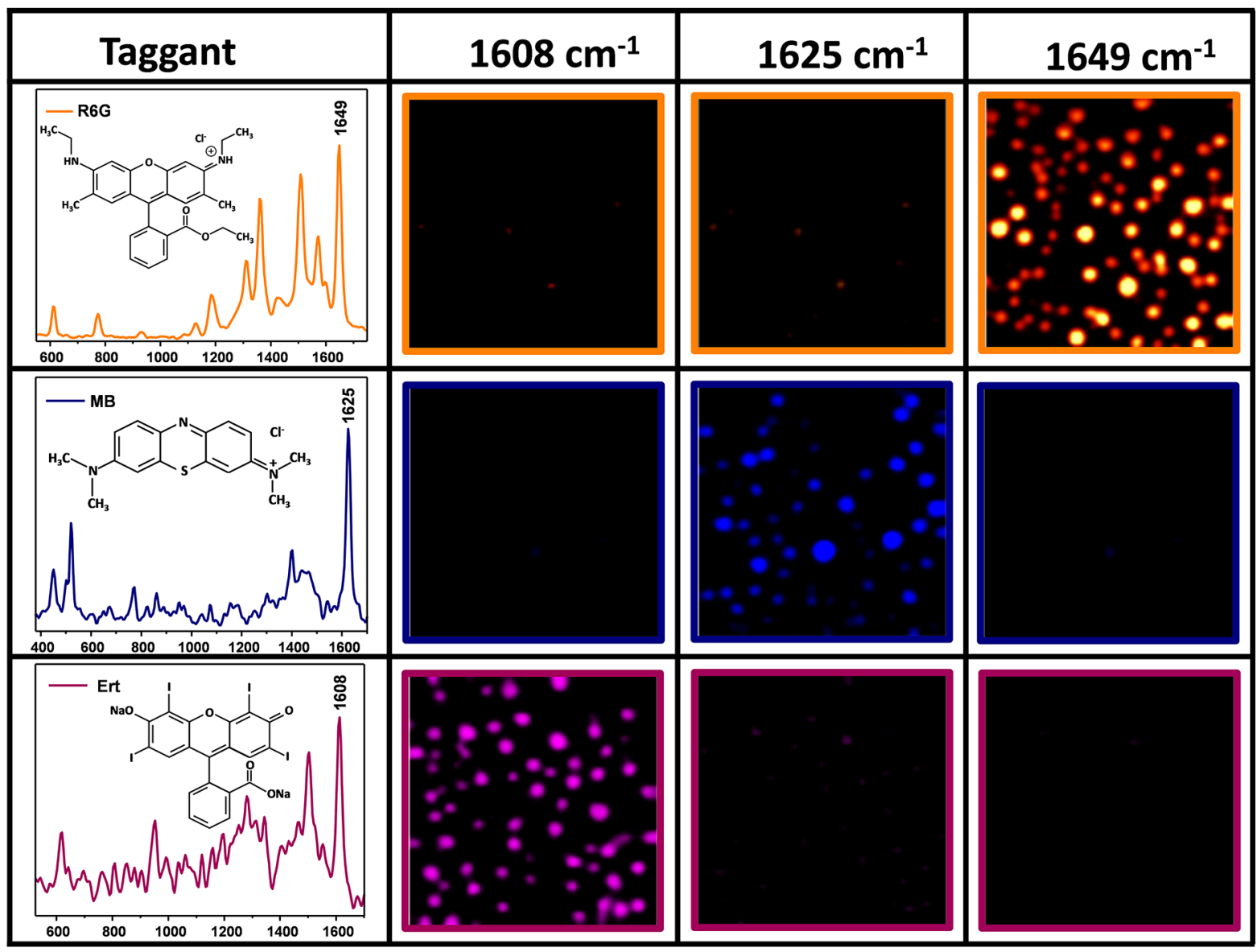

(b)

(c)
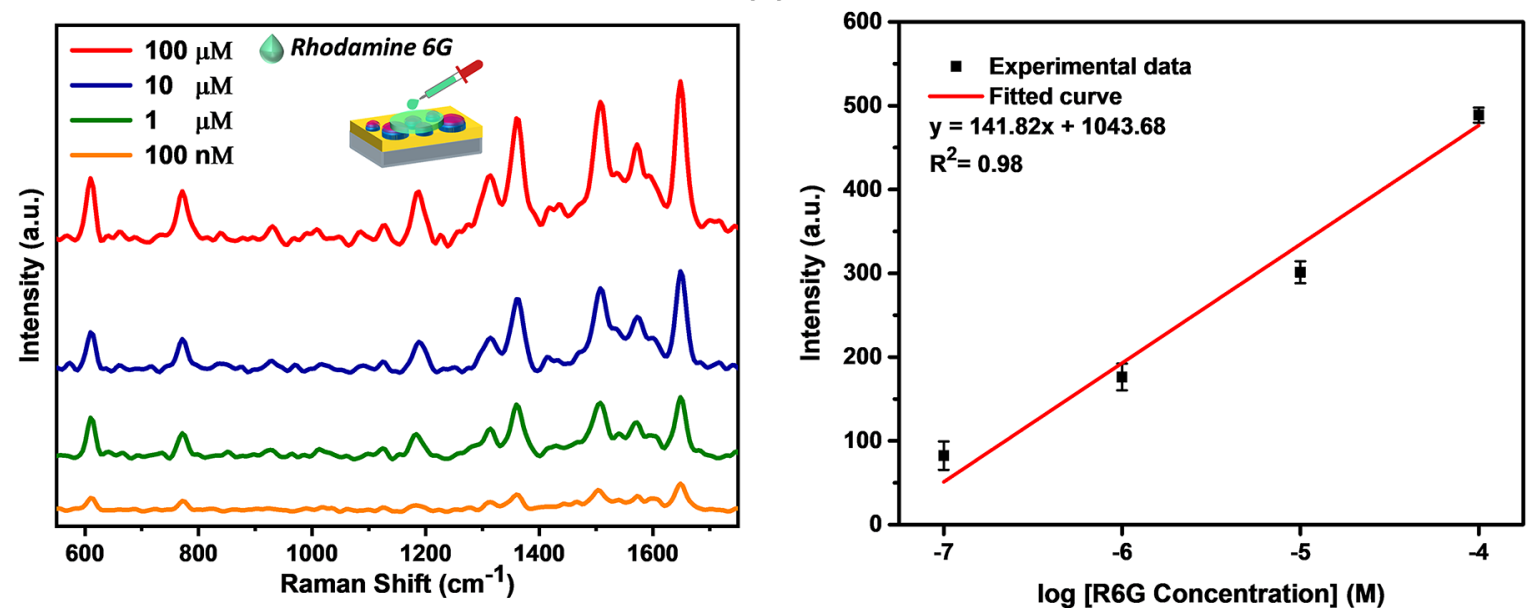

Figure 4. Molecular vibration- and SERS intensity-based security layer. (a) Raman intensity mapping images for three different samples prepared by deposition of R6G, MB, and Ert on the PUFs fabricated by dewetting of the P2VP film and assembly of colloidal gold nanoparticles. For each sample (row), the Raman intensity mapping was generated at three different Raman shifts specified at the top of the columns. (b,c) Variation in the SERS activity as a function of the concentration of the taggant molecule.

Figure 3 presents the authentication of the physically unclonable surfaces fabricated by dewetting of P2VP films. We first defined markers on top of the dewetted P2VP film through evaporation of copper from a stencil mask with an opening in the geometry of a cross-bar (Figure 3a). The marker was defined following the dewetting process; otherwise, the copper film would change the surface energy of the substrate in the regions covered by the markers. These markers allowed a true comparison of the spatial distribution of the dewetted features. Figure $3 \mathrm{~b}$ shows a series of images derived from the substrate following definition of the marker. These images were then binarized and converted into bit arrays (Figure 3c). For this purpose, the size of the binarized optical microscope image was first reduced from 400 pixels $\times 400$ pixels to 16 pixels $\times 16$ pixels. The resizing and image compression are commonly used to generate digitized keys 
from microscope images. ${ }^{9}$ Afterward, each image (16 pixels $\times$ 16 pixels) was then directly used to generate a security key that consists of 1-bits and 0-bits, which correspond to dewetted features and remaining regions, respectively. The resulting keys were 256-bits long. Figure $3 \mathrm{~d}$ schematically illustrates the authentication approach. The security keys are generated from different samples and stored in a database. For a sample of unknown authenticity, the processes of imaging, binarization, and size reduction are performed to generate a key, which is then compared with the keys present in the database. For the proof-of-concept demonstration, images from 11 different samples were used to construct 11 security keys (images are presented in Figures S5 and S6).

To probe the quality of the fabricated unclonable surfaces, we calculated uniqueness, randomness, reliability, and uniformity, which are figures of merit ${ }^{47}$ employed in PUF studies. The uniformity of the keys is a measure of the random distribution of " $0 \mathrm{~s}$ " and " $1 \mathrm{~s}$ " in a bit array. The uniformity of a key is simply obtained by the number of 1-bits divided by the total number of bits (see the Supporting Information for details). For a perfectly random process, the ideal value for the uniformity should be 0.5 , implying for equal distribution of 1 bits and 0-bits. ${ }^{47}$ The uniformity values (Figure 3e) of our keys ranged between 0.4688 and 0.6055 , with an arithmetic average of 0.5352 . Another metric is randomness, which is closely related to the uniformity. ${ }^{47}$ Among different measures of randomness, ${ }^{48}$ we used frequency test, which probes the equal distribution of 1-bits and 0-bits. The calculated $p$-value (see the Supporting Information for details) should be greater than 0.01 for a security key to be random. The majority $(9 / 11)$ of our keys resulted in $p$-values (Table $\mathrm{S} 1$ ) greater than 0.01 , implying the randomness of the fabricated patterns. It is known that the raw response of physical systems may be biased to an extent that depends on the physical nature of the process. ${ }^{49,50}$ In other words, there could be more instances of 0-bits or 1bits in the raw key. ${ }^{9}$ One approach to overcome this issue is classic von Neumann debiasing. ${ }^{51}$ Applying this debiasing approach significantly improved the uniformity and randomness of our keys. The $p$-values of the debiased keys were all random as evidenced with $p$-values greater than 0.01 .

The uniqueness is an important PUF parameter and measure of differentiating a key from a set of keys present in the database. The uniqueness is defined based on inter-chip Hamming distance $\left(\mathrm{HD}_{\text {INTER }}\right)$, which is simply the number of bits with a different response in comparing two distinct keys. ${ }^{47}$ The uniqueness is obtained by averaging $\mathrm{HD}_{\text {INTER }}$ among all different combinations of keys in a database. For 11 keys, for example, $H_{\text {INTER }}$ is calculated for $55(11 \times 10 / 2)$ combinations and then averaged over these combinations. The uniqueness was calculated as 0.4695 , a value that is close to the ideal value of 0.5 . The distribution of $\mathrm{HD}_{\text {INTER }}$ is given in Figure 3f. The reliability is a measure of the reproducibility of the response of a key to the same challenge performed under different operating conditions. The reliability is defined based on intra-chip Hamming distance $\left(\mathrm{HD}_{\text {INTRA }}\right)$. Ideally, the key should give an identical response to the same challenge and $\mathrm{HD}_{\text {INTRA }}$ should be zero, resulting in a reliability of $100 \%$. Figure $3 \mathrm{f}$ presents the distribution of $\mathrm{HD}_{\text {INTRA }}$ for a key challenged under five different conditions. Values of $\mathrm{HD}_{\text {INTRA }}$ are close to zero, showing the reliability of the presented system.

The optical microscopy images provide simple and rapid authentication of the PUFs with information limited to spatial distribution of the features. To enrich the information stored in the PUFs, we investigate Raman scattering of light due to molecular vibrations. One piece of information that is inherently encoded in the PUFs relates to the characteristic fingerprint vibrations of the dewetted polymer, P2VP in the present case. Mapping the intensity of the peak at $3057 \mathrm{~cm}^{-1}$, associated with the pyridine, visualizes the dewetted random features in Raman spectroscopy (Figure S7). The effective exploitation of Raman scattering in encoding requires the ability to incorporate taggant molecules of varying chemistries. ${ }^{40}$ An inherent challenge in incorporating taggant molecules over the PUFs is the weak inelastic scattering of light resulting in low levels of Raman intensities. The electromagnetic enhancement is the most widely utilized approach that typically takes advantage of formation of plasmonic hot spots in between closely spaced metallic nanostructures. ${ }^{52}$ For this purpose, we assembled colloidal gold NPs over the dewetted P2VP patterns, thanks to the pyridine group with strong affinity toward metallic nanoparticles. ${ }^{53}$ The assembled gold nanoparticles enable SERS from the taggant molecules and turn the dewetted features into SERS-active PUFs. To maximize the SERS activity from the dewetted features, we first investigated the assembly of citratestabilized gold nanoparticles for varying diameter and time. Among the explored conditions, the SERS activity was maximum when the diameter of gold NPs is $10 \mathrm{~nm}$ and the duration of the particle immobilization is $24 \mathrm{~h}$ (Figure S8). Varying the chemistry of the probe molecule is a highly effective means of increasing the complexity of the security label. The molecular structure of the probe molecule determines the position of the characteristic fingerprint vibrations. Figure 4a presents results obtained using three different taggant molecules: rhodamine 6G (R6G), methylene blue (MB), and erythrosine (Ert). The key point is that Raman mappings display the PUFs only when choosing the Raman shift position that matches the characteristic fingerprint vibration of the taggant molecule. These results show the integration of spectral and spatial information in the same platform. Note that the spatial positions of features derived from the same position of the substrate using optical microscopy and Raman mappings are not necessarily identical (Figure S9). This contrast arises from the dependence of the Raman intensity on the formation of plasmonic hot spots, which varies depending on the lateral and out-of-plane dimensions of the dewetted features.

The intensity of the Raman scattering can be programmed, providing a convenient pathway for integrating additional information to the PUFs. The intensity of the characteristic Raman bands scales with the concentration of the taggant molecule. Figure $4 b$, for example, presents SERS spectra for R6G deposited from solutions with concentrations that range from $100 \mu \mathrm{M}$ to $100 \mathrm{nM}$. The Raman bands at 1362,1507 , and $1649 \mathrm{~cm}^{-1}$ associated with the aromatic $\mathrm{C}-\mathrm{C}$ stretching vibrations all become more intense with the increasing concentration of R6G. Figure 4c presents the Raman scattering intensity at a position of $1649 \mathrm{~cm}^{-1}$ as a function of the concentration of R6G. The relation between the intensity (I) and concentration $(C)$ can be well described with the equation $I=141.82 C+1043.68$. The dependence of the Raman scattering intensity on the solution concentration of the probe molecule suggests that the levels of the SERS activity can be used as an additional security layer. This type of security can be applied based on setting a threshold intensity level, below 


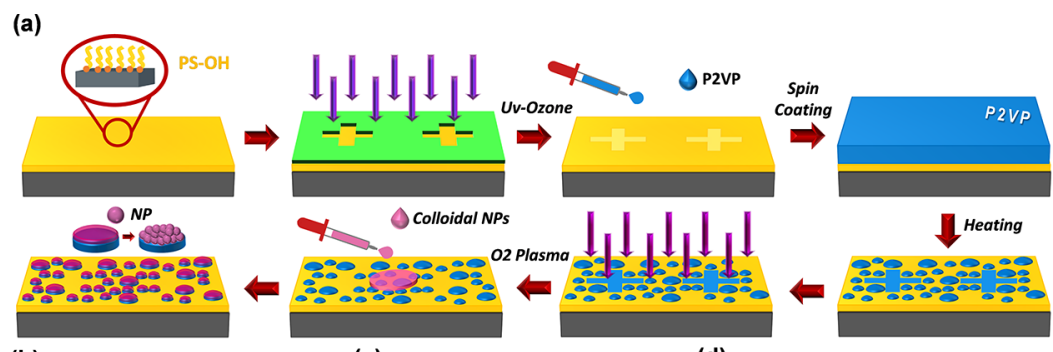

(b)

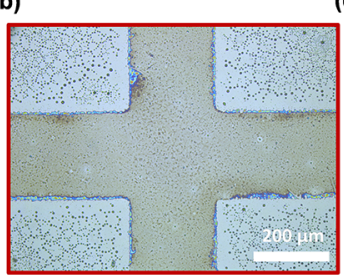

(e)

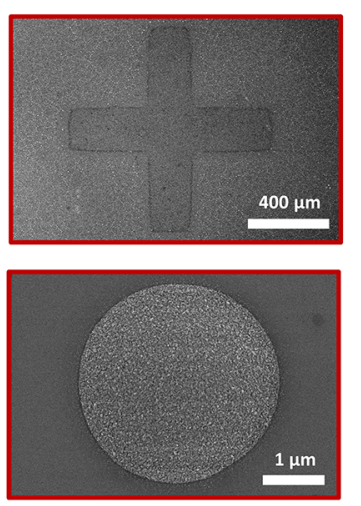

(c)

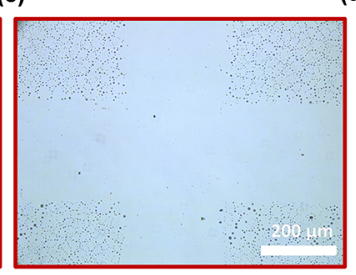

(d)

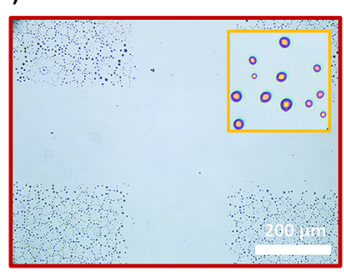

(f)

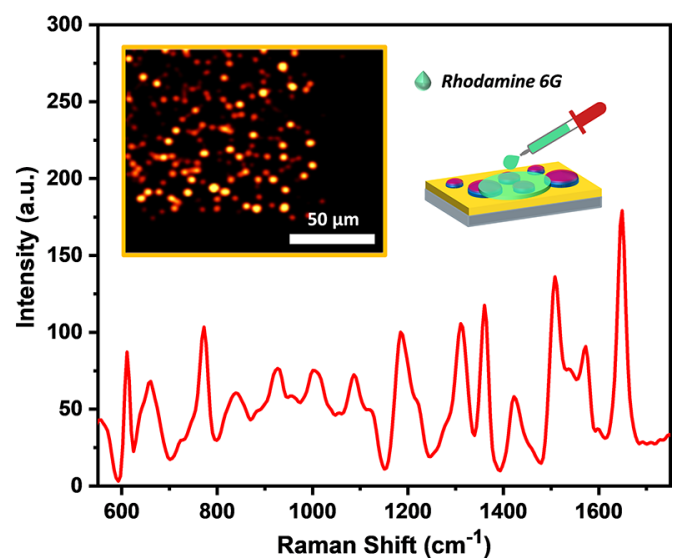

Figure 5. Patterning of PUFs. (a) Schematic illustration of the patterning process. (b-d) Optical microscopy images of the patterned region following (b) dewetting, (c) oxygen plasma treatment, and (d) assembly of colloidal gold NPs. (e) SEM images of the patterned cross-bar and an individual dewetted feature. (f) SERS spectrum obtained on the dewetted features. The inset presents a Raman mapping image generated based on the characteristic vibration of $\mathrm{R} 6 \mathrm{G}$ at $1649 \mathrm{~cm}^{-1}$.

which the authenticity is not confirmed. In more complex authentication configurations, the level of intensity from individual features can also be encoded.

The incorporation of the fabricated PUFs into products may require control over their dimensions and positions. We used localized surface energy modification together with oxygen plasma etching to generate PUFs within patterned regions (Figure 5a). The first step of fabrication was masked UVozone treatment of the PS-grafted substrates. The exposure to UV-ozone resulted in the hydrophilization and removal of PS, changing the wetting behavior from hydrophobic to hydrophilic. Spin-coating a film of P2VP and thermal annealing were then performed as in the case of unpatterned samples. The thermal annealing resulted in dewetting of the P2VP film only in regions that were masked during $\mathrm{UV}$-ozone treatment (Figure $5 b$ ). This experiment clearly illustrated the importance of the surface wetting properties for the dewetting process. Following the dewetting of the polymer film, we performed a brief oxygen plasma treatment to the substrate. This oxygen plasma treatment only removed the P2VP films that did not dewet. This selective removal ability emerges from the large height contrast between the spin-coated film with a thickness of $\sim 25 \mathrm{~nm}$ versus dewetted features with a height that ranges from 160 to $560 \mathrm{~nm}$. Optical microscopy images following oxygen plasma treatment (Figure 5c) confirm that dewetted features are confined in regions defined by the mask used in the UV-ozone treatment (see Figure S10 for more images). Despite oxygen plasma treatment, dewetted P2VP features remain functional and can successfully immobilize colloidal gold NPs (Figure 5d-f). The patterned substrate exhibited SERS activity and the characteristic peaks of the taggant can be clearly detected (Figure $5 \mathrm{f}$ ). The intensity of the characteristic peaks on the patterned PUFs (188) was slightly lower in comparison with that of the unpatterned (489) substrates. This contrast is most likely the result of the reduced number of gold NPs (see Figure S11) on the patterned PUFs. The decrease in the number of adsorbed gold NPs may relate to the increased planarity of the oxygen plasma-treated dewetted features.

Practical applications require fabrication of security labels on transparent and flexible substrates. Figure $6 \mathrm{a}-\mathrm{c}$ presents such capabilities and demonstrates that the materials and methods presented in this study can be readily adapted to different substrates. The glass resembles the same silicon dioxide chemistry allowing for direct grafting of PS chains as in the case of silicon substrates. The formation of dewetted patterns and assembly of colloidal gold NPs on glass following the similar processes resulted in SERS-active PUFs on transparent substrates. One particular difference is the color of the dewetted features following assembly of gold NPs due to the different reflection properties of silicon and glass substrates. As 
(a)

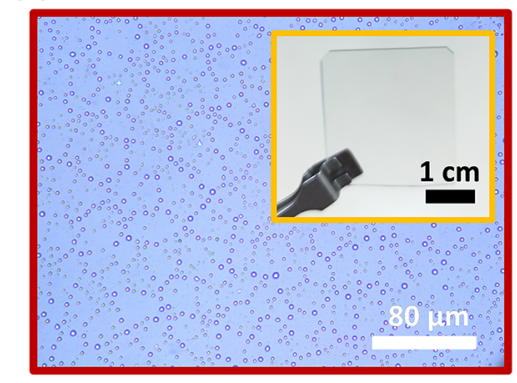

(b)

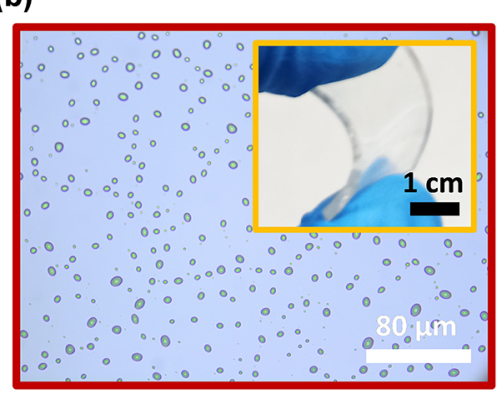

(d)

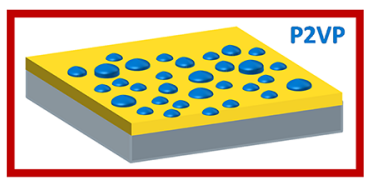

Store for 1 year

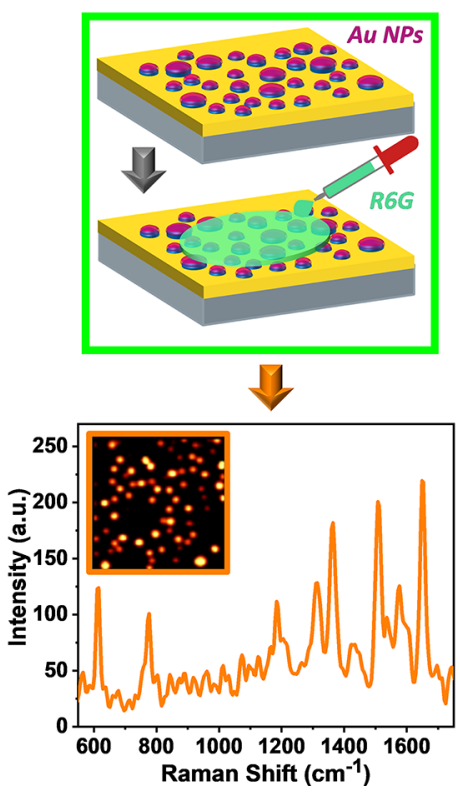

(c)

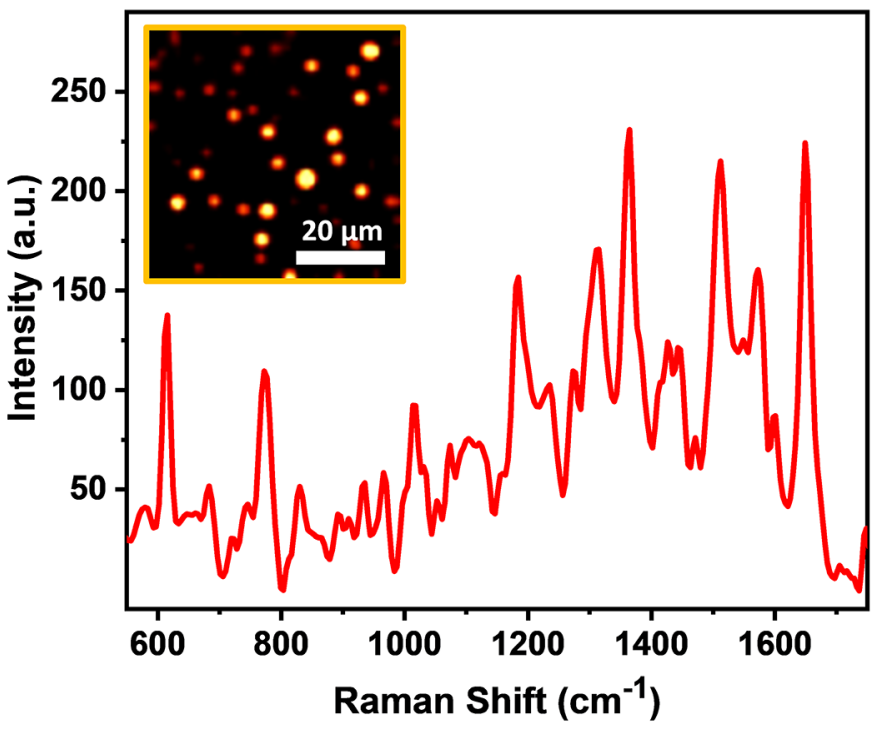

(e)

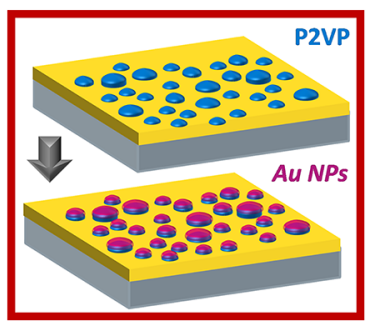

Store for 1 year

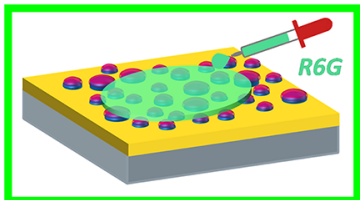

I

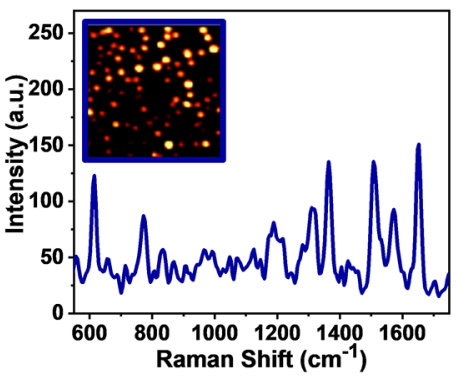

(f)

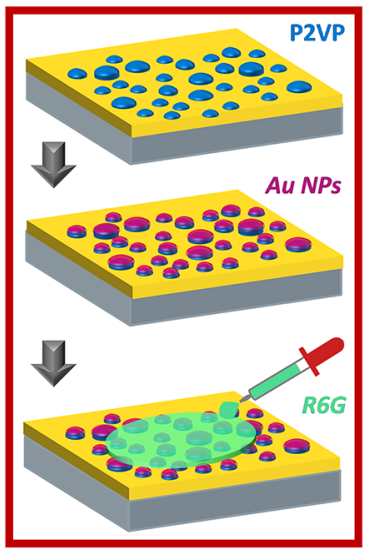

Store for 1 year

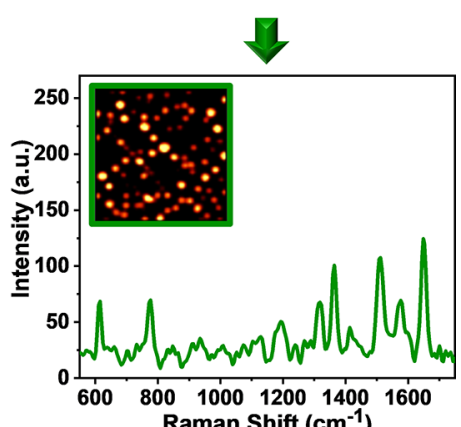

Figure 6. Different types of substrates and stability. $(a-c)$ Fabrication of PUFs on transparent and flexible substrates. (a) Glass and (b) PDMS substrates. $(\mathrm{a}, \mathrm{b})$ Optical microscopy images of the randomly positioned P2VP features. The insets are photographs of the samples. (c) Raman spectrum of $100 \mu \mathrm{M}$ R6G deposited on the assembled gold NPs on the glass substrate. The inset presents a mapping image generated based on Raman scattering at a position of $1649 \mathrm{~cm}^{-1}$. (d-f) Stability of the PUFs. Stability after (d) dewetting of P2VP film, (e) assembly of gold NPs, and (f) deposition of the taggant. In each case, the substrate was stored in air for 1 year. The concentration of R6G was $100 \mu \mathrm{M}$. Raman spectra and Raman mapping images based on Raman scattering at a position of $1649 \mathrm{~cm}^{-1}$ are given at the bottom.

flexible substrates, we used drop-cast films of polydimethylsiloxane (PDMS). Following oxygen plasma and UV-ozone treatment, polar silanol groups are generated on the surface of PDMS. These silanol groups enable grafting of PS to tune the surface energy for the dewetting. PUFs can be successfully fabricated on flexible substrates and can be validated using optical microscopy and Raman mapping images (see Figure S12). PUFs on the glass and PDMS substrate were remarkably SERS active as demonstrated by clear spectra of the taggant; however, the maximum intensity of the characteristic peak was 
slightly lower on glass (224) and PDMS (133) substrates than that on silicon (489) substrates. We suggest that this difference arises from the size distribution of the dewetted features and impact of this distribution on the assembly of colloidal gold NPs and their SERS activity.

The stability of the PUFs is significantly important for practical implementation in real-world products. The stability of the fabricated PUFs was investigated at three different levels by storing the surfaces for 1 year following dewetting of the P2VP film, assembly of gold NPs, and deposition of the taggant molecule. Figure $6 \mathrm{~d}-\mathrm{f}$ presents schematic descriptions together with the SERS spectra of R6G at the end of the process. The dewetted P2VP film was remarkably stable for at least one year. The dewetted P2VP features did not exhibit any loss in their visibility under optical microscopy, confirming the stability of the first security layer. Note that in some applications (e.g., electronics), this first security layer can be permanently integrated to the product by transferring the random patterns to the underlying substrate by etching processes. $^{54}$ The dewetted P2VP features remained functional after storage for 1 year and gold NPs could be successfully adsorbed with a high binding density (see Figure S13 for SEM images). The substrate exhibited significant SERS activity following deposition of the taggant molecule. The SERS intensity of the characteristic vibration band of R6G was $~ 220$, which was slightly lower than the intensity obtained on the fresh sample ( 489). The intensity of Raman scattering from the sub-micron features was found to be lower for the samples stored for one year in comparison with that of the fresh sample. In the case of stability after assembly of gold NPs and deposition of the taggant, the characteristic vibration bands of the taggant could be clearly observed with the intensities of $\sim 150$ and $\sim 120$, respectively. The absence of a significant difference in the morphology of the features as imaged via SEM and optical microscopy (Figure S13) suggests that contamination from the ambient together with deterioration of the taggant molecule can contribute to slight decay of the SERS activity. Overall, these results suggest a remarkable level of stability in the presented PUFs. Further improvements in the stability can be provided using Raman invisible encapsulation layers and engineering the interaction between the taggant molecule and nanoparticles. The robust nature of the presented platform also allows easy cleaning of the surfaces in the case of contamination. The optical microscopy and Raman mapping images (Figure S14) obtained from the same region of the substrate show that the surface retains its functionality after contamination with dust particles and cleaning in water under sonication.

\section{CONCLUSIONS}

This study has demonstrated a new approach in fabrication of encoded surfaces that are derived from the combined use of dewetting instabilities in polymer thin films and self-assembly of colloidal nanomaterials. The stochastic nature of the processes of polymer dewetting together with the ability to position functional moieties in confined regions enables assembly of colloidal nanomaterials into features with random positions and sizes with separation distances in the micrometer length scale, making the identification with optical techniques viable. The strong and selective interaction between nanoscale building blocks and randomly positioned functional features is key in fabrication of robust and contamination-free surfaces, leading to images with low levels of noise. The fabricated security labels can withstand sonication in water and demonstrate stability for a period greater than one year. The simple processes that are described in this study are compatible with conventional fabrication methods, suggesting their implementation with deterministic security labels using massively parallel fabrication routes. The seamless integration with colloidal nanomaterials offers an unlimited number of options in increasing the complexity of the encoded surfaces and allowing identification with different methods possible. The complexity of the dewetted features can be further elaborated using structured polymers. ${ }^{55,56}$ The authentication of the fabricated unclonable surfaces using mobile devices ${ }^{57}$ and advanced image-processing techniques ${ }^{58,59}$ appears promising for future studies. Rapid and easy fabrication of threedimensional functional features over large areas may serve as a valuable platform for self-assembly of colloidal nanomaterials on non-planar surfaces.

\section{EXPERIMENTAL METHODS}

Materials. Silicon wafers $(\langle 100\rangle, \mathrm{N} / \mathrm{Phos})$ were purchased from Wafer World Inc. Glass slides were purchased from Isolab Inc. Hydroxyl-terminated PS (PS-OH, $M_{\mathrm{n}}=22.0 \mathrm{~kg} / \mathrm{mol}, \mathrm{PDI}=1.06$ ) and P2VP $\left(M_{\mathrm{n}}=28.0 \mathrm{~kg} / \mathrm{mol}\right.$, PDI = 1.09 $)$ were purchased from Polymer Source Inc. PDMS (Sylgard 184 kit) was purchased from Dow Corning. Chlorobenzene, toluene, and diiodomethane were purchased from Sigma-Aldrich. Ethanol, formamide, and ethylene glycol were purchased from Merck. R6G $\left(\mathrm{C}_{28} \mathrm{H}_{31} \mathrm{~N}_{2} \mathrm{O}_{3} \mathrm{Cl}, M_{\mathrm{n}}=\right.$ $479.01 \mathrm{~g} / \mathrm{mol}), \mathrm{MB}\left(\mathrm{C}_{16} \mathrm{H}_{18} \mathrm{ClN}_{3} \mathrm{~S}, M_{\mathrm{n}}=319.85 \mathrm{~g} / \mathrm{mol}\right)$, and Ert $\left(\mathrm{C}_{20} \mathrm{H}_{6} \mathrm{I}_{4} \mathrm{Na}_{2} \mathrm{O}_{5}, M_{\mathrm{n}}=879.86 \mathrm{~g} / \mathrm{mol}\right)$ were purchased from SigmaAldrich. Copper pellets (99.99\% purity) were purchased from Nanovak Inc. Citrate-stabilized gold NPs $(10,20$, and $40 \mathrm{~nm}$ in diameter) were purchased from Ted Pella Inc.

Substrate Modification with an End-Grafted PS Layer. The silicon substrates were cut in $1 \times 1 \mathrm{~cm}^{2}$ dimensions and washed under sonication in ethyl alcohol for $5 \mathrm{~min}$. The substrates were then exposed to UV-ozone for $30 \mathrm{~min}$. After this process, the freshly prepared solution of hydroxyl-terminated PS was spin-coated (4000 rpm) from a 2 wt \% solution in toluene. This film was heated in a glovebox for $5 \mathrm{~min}$ at $250{ }^{\circ} \mathrm{C}$. After heating, the excess material was removed by repeated washing in toluene. The substrates modified with the end-grafted PS layer were revealed by drying with nitrogen. The end-grafting processes were same on the glass and PDMS substrates. The latter substrate was prepared by pouring PDMS and curing agent (10:1) over a Petri dish.

Fabrication of Unclonable Surfaces through Dewetting of P2VP. A film of P2VP was spin-coated (4000 rpm) on top of the endgrafted PS layer. For this purpose, we prepared solutions of P2VP in chlorobenzene. Unless otherwise stated, the solutions contained $1 \mathrm{wt}$ $\%$ P2VP. The dewetting was induced by thermal annealing in a glovebox for $5 \mathrm{~min}$. Unless otherwise stated, the temperature of annealing was $250{ }^{\circ} \mathrm{C}$. The preparation of dewetted P2VP films followed the same processes on the glass and PDMS substrates.

Self-Assembly of Colloids on the Unclonable Surfaces. Citrate-stabilized colloidal NPs with a volume of $150 \mu \mathrm{L}$ were drop casted on the dewetted P2VP features spanning an area of $1 \mathrm{~cm} \times 1$ $\mathrm{cm}$. Unless otherwise stated, the gold NP treatment time was $24 \mathrm{~h}$ and the diameter of the gold NPs was $10 \mathrm{~nm}$. Afterward, the substrate was washed in water under sonication for $3 \mathrm{~min}$ and dried with nitrogen.

Fabrication of the Marker for Image Analysis. A stencil mask with openings in the shape of a cross-bar was fabricated through laser writing. A $\sim 50 \mathrm{~nm}$ film of copper was vapor deposited on the unclonable surfaces through the stencil mask. The deposition was performed using a thermal evaporator (Nanovak, NVTS-500) at $30 \mathrm{~V}$ and $10 \mathrm{~A}$ with a base pressure of $10^{-8}$ Torr. The process pressure was $5 \times 10^{-6}$ Torr, and the deposition rate was $0.04 \mathrm{~nm} / \mathrm{s}$.

Patterning of PUFs. The stencil mask was placed over the substrate modified with a layer of end-grafted PS. UV-ozone treatment was performed through the stencil mask for $10 \mathrm{~min}$. 
Immediately after the UV-ozone treatment, the entire substrate was spin-coated with a film of P2VP ( 1 wt \% in chlorobenzene at 4000 $\mathrm{rpm}$ ) and thermally annealed in a glovebox at $250{ }^{\circ} \mathrm{C}$ for $5 \mathrm{~min}$. The entire substrate was then subjected to oxygen plasma (Harrick plasma cleaner PDC-32G-2) treatment for $15 \mathrm{~min}$. The plasma power was kept low with an oxygen flow rate of $\sim 5 \mathrm{sccm}$ and pressure of $\sim 100$ $\mathrm{mT}$.

Image Analysis. The analysis of images was performed using ImageJ and MATLAB software. The optical microscopy and Raman mapping images were binarized in black and white image format. The binarization leads to black-colored dewetted features in a white background. The coordinates of the features with respect to the marker were then extracted from the software. These coordinates were utilized in generating keys consisting of 0-bits and 1-bits.

Characterization. The thickness of the polymer films was measured using an ellipsometer (Gaertner LSE Stokes). Water contact angle and surface energy measurements were performed using an optical tensiometer (Attension Theta Lite). The morphology of the substrates was analyzed using a scanning electron microscope (Zeiss EVO LS10) operated at $25 \mathrm{kV}$. The topography of the surfaces was analyzed with AFM (Veeco Multimode 8) in the tapping mode. All optical microscope images were taken using the Lecia DM750M.

All Raman mapping images were recorded using an excitation laser with a wavelength of $532 \mathrm{~nm}$ and a power of $3.2 \mathrm{~mW}$ via a confocal Raman spectrometer (WITec Alpha300 M+) equipped with finefocusing microscope objectives. Before obtaining Raman spectra, the spectrometer was calibrated using the Raman band of the silicon wafer at $520 \mathrm{~cm}^{-1}$. We used three different taggant molecules: R6G, MB, and Ert. Aqueous solutions of taggant molecules with a volume of 1 $\mu \mathrm{L}$ were spotted on the substrate. Following evaporation of water at room temperature, the substrate was placed under the objective. The SERS mappings were generated on $50 \times 50 \mu \mathrm{m}^{2}$ areas with a $1 \mu \mathrm{m}$ step size using a $100 \times$ objective $(\mathrm{NA}=0.90)$ by integrating the characteristic peaks at 1649,1625 , and $1608 \mathrm{~cm}^{-1}$ for R6G, MB, and Ert, respectively. All spectra were baseline-subtracted.

\section{ASSOCIATED CONTENT}

\section{s) Supporting Information}

The Supporting Information is available free of charge at https://pubs.acs.org/doi/10.1021/acsami.0c16846.

Surface energy measurements, effect of the thickness of the polymer film on the surface wetting properties and size distribution of dewetted features, additional images used for the construction of the database, Raman mapping and images of the dewetted P2VP film, effect of the diameter of gold nanoparticles and deposition time on the SERS characteristics, and additional optical microscopy and SEM images (PDF)

\section{AUTHOR INFORMATION}

\section{Corresponding Author}

M. Serdar Onses - ERNAM-Nanotechnology Research and Application Center, Erciyes University, Kayseri 38039, Turkey; Department of Materials Science and Engineering, Erciyes University, Kayseri 38039, Turkey; UNAM-Institute of Materials Science and Nanotechnology, Bilkent University, Ankara 06800, Turkey; (1) orcid.org/0000-0001-68987700; Email: onses@erciyes.edu.tr

\footnotetext{
Authors

Neslihan Torun - ERNAM-Nanotechnology Research and Application Center, Erciyes University, Kayseri 38039, Turkey

Ilker Torun - ERNAM-Nanotechnology Research and Application Center, Erciyes University, Kayseri 38039,
}

Turkey

Menekse Sakir - ERNAM-Nanotechnology Research and Application Center, Erciyes University, Kayseri 38039, Turkey; O orcid.org/0000-0003-3102-0947

Mustafa Kalay - ERNAM-Nanotechnology Research and Application Center, Erciyes University, Kayseri 38039, Turkey; Department of Electricity and Energy, Kayseri University, Kayseri 38039, Turkey

Complete contact information is available at: https://pubs.acs.org/10.1021/acsami.0c16846

\section{Notes}

The authors declare no competing financial interest.

\section{ACKNOWLEDGMENTS}

We thank Chi-Chun Liu for valuable discussions on the analysis and processing of the images. This work was supported by the Research Fund of the Erciyes University (project number FDS-2020-9706). M.S.O. acknowledges partial support from The Science Academy, Turkey through the Young Scientist Award Program.

\section{REFERENCES}

(1) McGrath, T.; Bagci, I. E.; Wang, Z. M.; Roedig, U.; Young, R. J. A PUF Taxonomy. Appl. Phys. Rev. 2019, 6, 011303.

(2) Maes, R.; Verbauwhede, I. Physically Unclonable Functions: A study on the State of the Art and Future Research Directions. In Towards Hardware-Intrinsic Security; Sadeghi, A.-R., Naccache, D., Eds.; Information Security and Cryptography; Springer: Berlin, Heidelberg, 2010; pp 3-37.

(3) Pappu, R.; Recht, B.; Taylor, J.; Gershenfeld, N. Physical OneWay Functions. Science 2002, 297, 2026-2030.

(4) Arppe, R.; Sørensen, T. J. Physical Unclonable Functions Generated Through Chemical Methods for Anti-Counterfeiting. Nat. Rev. Chem. 2017, 1, 0031.

(5) Shikha, S.; Salafi, T.; Cheng, J.; Zhang, Y. Versatile Design and Synthesis of Nano-Barcodes. Chem. Soc. Rev. 2017, 46, 7054-7093.

(6) Yoon, B.; Lee, J.; Park, I. S.; Jeon, S.; Lee, J.; Kim, J.-M. Recent Functional Material Based Approaches to Prevent and Detect Counterfeiting. J. Mater. Chem. 2013, 1, 2388-2403.

(7) Kim, J.; Yun, J. M.; Jung, J.; Song, H.; Kim, J.-B.; Ihee, H. AntiCounterfeit Nanoscale Fingerprints Based on Randomly Distributed Nanowires. Nanotechnology 2014, 25, 155303.

(8) Smith, A. F.; Patton, P.; Skrabalak, S. E. Plasmonic Nanoparticles as a Physically Unclonable Function for Responsive Anti-Counterfeit Nanofingerprints. Adv. Funct. Mater. 2016, 26, 1315-1321.

(9) Leem, J. W.; Kim, M. S.; Choi, S. H.; Kim, S.-R.; Kim, S.-W.; Song, Y. M.; Young, R. J.; Kim, Y. L. Edible Unclonable Functions. Nat. Commun. 2020, 11, 328.

(10) Liu, Y.; Han, F.; Li, F.; Zhao, Y.; Chen, M.; Xu, Z.; Zheng, X.; Hu, H.; Yao, J.; Guo, T. Inkjet-Printed Unclonable Quantum Dot Fluorescent Anti-Counterfeiting Labels with Artificial Intelligence Authentication. Nat. Commun. 2019, 10, 2409.

(11) Tian, L.; Liu, K.-K.; Fei, M.; Tadepalli, S.; Cao, S.; Geldmeier, J. A.; Tsukruk, V. V.; Singamaneni, S. Plasmonic Nanogels for Unclonable Optical Tagging. ACS Appl. Mater. Interfaces 2016, 8, 4031-4041.

(12) Carro-Temboury, M. R.; Arppe, R.; Vosch, T.; Sørensen, T. J. An Optical Authentication System Based on Imaging of ExcitationSelected Lanthanide Luminescence. Sci. Adv. 2018, 4, No. e1701384.

(13) Hu, Z.; Comeras, J. M. M. L.; Park, H.; Tang, J.; Afzali, A.; Tulevski, G. S.; Hannon, J. B.; Liehr, M.; Han, S.-J. Physically Unclonable Cryptographic Primitives Using Self-Assembled Carbon Nanotubes. Nat. Nanotechnol. 2016, 11, 559-565. 
(14) Alharbi, A.; Armstrong, D.; Alharbi, S.; Shahrjerdi, D. Physically Unclonable Cryptographic Primitives by Chemical Vapor Deposition of Layered $\mathrm{MoS}_{2}$. ACS Nano 2017, 11, 12772-12779.

(15) Zhang, R.; Jiang, H.; Wang, Z. R.; Lin, P.; Zhuo, Y.; Holcomb, D.; Zhang, D. H.; Yang, J. J.; Xia, Q. Nanoscale Diffusive Memristor Crossbars as Physical Unclonable Functions. Nanoscale 2018, 10, 2721-2726.

(16) Bae, H. J.; Bae, S.; Park, C.; Han, S.; Kim, J.; Kim, L. N.; Kim, K.; Song, S.-H.; Park, W.; Kwon, S. Biomimetic Microfingerprints for Anti-Counterfeiting Strategies. Adv. Mater. 2015, 27, 2083-2089.

(17) Ma, T.; Li, T.; Zhou, L.; Ma, X.; Yin, J.; Jiang, X. Dynamic Wrinkling Pattern Exhibiting Tunable Fluorescence for Anticounterfeiting Applications. Nat. Commun. 2020, 11, 1811.

(18) Reiter, G. Dewetting of Thin Polymer Films. Phys. Rev. Lett. 1992, 68, 75 .

(19) Xu, L.; Shi, T.; An, L. The Dewetting Dynamics of the Polymer Thin Film by Solvent Annealing. J. Chem. Phys. 2008, 129, 044904.

(20) Mukherjee, R.; Das, S.; Das, A.; Sharma, S. K.; Raychaudhuri, A. K.; Sharma, A. Stability and Dewetting of Metal Nanoparticle Filled Thin Polymer Films: Control of Instability Length Scale and Dynamics. ACS Nano 2010, 4, 3709-3724.

(21) Tan, C. K.; Blackwood, D. J. Corrosion Protection by Multilayered Conducting Polymer Coatings. Corros. Sci. 2003, 45, 545-557.

(22) Onses, M. S.; Wan, L.; Liu, X.; Kiremitler, N. B.; Yilmaz, H.; Nealey, P. F. Self-Assembled Nanoparticle Arrays on Chemical Nanopatterns Prepared Using Block Copolymer Lithography. ACS Macro Lett. 2015, 4, 1356-1361.

(23) Zhang, D.; Tong, J.; Xia, B. Humidity-Sensing Properties of Chemically Reduced Graphene Oxide/Polymer Nanocomposite Film Sensor Based on Layer-by-Layer Nano Self-Assembly. Sens. Actuators, B 2014, 197, 66-72.

(24) He, Z.; Xiao, B.; Liu, F.; Wu, H.; Yang, Y.; Xiao, S.; Wang, C.; Russell, T. P.; Cao, Y. Single-Junction Polymer Solar Cells with High Efficiency and Photovoltage. Nat. Photonics 2015, 9, 174-179.

(25) Cai, Y.; Newby, B.-m. Z. Dewetting of Polystyrene Thin Films on Poly(ethylene glycol)-Modified Surfaces as a Simple Approach for Patterning Proteins. Langmuir 2008, 24, 5202-5208.

(26) Gentili, D.; Foschi, G.; Valle, F.; Cavallini, M.; Biscarini, F. Applications of Dewetting in Micro and Nanotechnology. Chem. Soc. Rev. 2012, 41, 4430-4443.

(27) Wang, J. Z.; Zheng, Z. H.; Li, H. W.; Huck, W. T. S.; Sirringhaus, H. Dewetting of Conducting Polymer Inkjet Droplets on Patterned Surfaces. Nat. Mater. 2004, 3, 171-176.

(28) Yoon, B.; Acharya, H.; Lee, G.; Kim, H.-C.; Huh, J.; Park, C. Nanopatterning of Thin Polymer Films by Controlled Dewetting on a Topographic Pre-Pattern. Soft Matter 2008, 4, 1467-1472.

(29) Mukherjee, R.; Bandyopadhyay, D.; Sharma, A. Control of Morphology in Pattern Directed Dewetting of Thin Polymer Films. Soft Matter 2008, 4, 2086-2097.

(30) Bystrenova, E.; Facchini, M.; Cavallini, M.; Cacace, M. G.; Biscarini, F. Multiple Length-Scale Patterning of DNA by StampAssisted Deposition. Angew. Chem. 2006, 118, 4897-4900.

(31) Ram, S.; Ward, E. S.; Ober, R. J. Beyond Rayleigh's Criterion: A Resolution Measure with Application to Single-Molecule Microscopy. Proc. Natl. Acad. Sci. U. S. A. 2006, 103, 4457-4462.

(32) Langer, J.; Jimenez de Aberasturi, D.; Aizpurua, J.; AlvarezPuebla, R. A.; Auguié, B.; Baumberg, J. J.; Bazan, G. C.; Bell, S. E. J.; Boisen, A.; Brolo, A. G.; Choo, J.; Cialla-May, D.; Deckert, V.; Fabris, L.; Faulds, K.; García de Abajo, F. J.; Goodacre, R.; Graham, D.; Haes, A. J.; Haynes, C. L.; et al. Present and Future of Surface-Enhanced Raman Sattering. ACS Nano 2020, 14, 28-117.

(33) Pekdemir, S.; Torun, I.; Sakir, M.; Ruzi, M.; Rogers, J. A.; Onses, M. S. Chemical Funneling of Colloidal Gold Nanoparticles on Printed Arrays of End-Grafted Polymers for Plasmonic Applications. ACS Nano 2020, 14, 8276-8286.

(34) Cui, Y.; Hegde, R. S.; Phang, I. Y.; Lee, H. K.; Ling, X. Y. Encoding Molecular Information in Plasmonic Nanostructures for Anti-Counterfeiting Applications. Nanoscale 2014, 6, 282-288.
(35) Cui, Y.; Phang, I. Y.; Lee, Y. H.; Lee, M. R.; Zhang, Q.; Ling, X. Y. Multiplex Plasmonic Anti-Counterfeiting Security Labels Based on Surface-Enhanced Raman Scattering. Chem. Commun. 2015, 51, 5363-5366.

(36) Li, D.; Tang, L.; Wang, J.; Liu, X.; Ying, Y. Multidimensional SERS Barcodes on Flexible Patterned Plasmonic Metafilm for Anticounterfeiting Applications. Adv. Opt. Mater. 2016, 4, 14751480.

(37) Zhou, Y.; Zhao, G.; Bian, J.; Tian, X.; Cheng, X.; Wang, H.; Chen, H. Multiplexed SERS Barcodes for Anti-Counterfeiting. ACS Appl. Mater. Interfaces 2020, 12, 28532-28538.

(38) Liu, Y.; Lee, Y. H.; Lee, M. R.; Yang, Y.; Ling, X. Y. Flexible Three-Dimensional Anticounterfeiting Plasmonic Security Labels: Utilizing Z-Axis-Dependent SERS Readouts to Encode Multilayered Molecular Information. ACS Photonics 2017, 4, 2529-2536.

(39) Zheng, Y.; Jiang, C.; Ng, S. H.; Lu, Y.; Han, F.; Bach, U.; Gooding, J. J. Unclonable Plasmonic Security Labels Achieved by Shadow-Mask-Lithography-Assisted Self-Assembly. Adv. Mater. 2016, 28, 2330-2336.

(40) Gu, Y.; He, C.; Zhang, Y.; Lin, L.; Thackray, B. D.; Ye, J. GapEnhanced Raman Tags for Physically Unclonable Anticounterfeiting Labels. Nat. Commun. 2020, 11, 516.

(41) Pekdemir, S.; Ipekci, H. H.; Serhatlioglu, M.; Elbuken, C.; Onses, M. S. SERS-Active Linear Barcodes by Microfluidic-Assisted Patterning. J. Colloid Interface Sci. 2021, 584, 11-18.

(42) Torun, I.; Celik, N.; Hancer, M.; Es, F.; Emir, C.; Turan, R.; Onses, M. S. Water Impact Resistant and Antireflective Superhydrophobic Surfaces Fabricated by Spray Coating of Nanoparticles: Interface Engineering via End-Grafted Polymers. Macromolecules 2018, 51, 10011-10020.

(43) Malynych, S.; Luzinov, I.; Chumanov, G. Poly(vinyl pyridine) as a Universal Surface Modifier for Immobilization of Nanoparticles. J. Phys. Chem. B 2002, 106, 1280-1285.

(44) Kiremitler, N. B.; Torun, I.; Altintas, Y.; Patarroyo, J.; Demir, H. V.; Puntes, V. F.; Mutlugun, E.; Onses, M. S. Writing Chemical Patterns Using Electrospun Fibers as Nanoscale Inkpots for Directed Assembly of Colloidal Nanocrystals. Nanoscale 2020, 12, 895-903.

(45) Xue, L.; Han, Y. Pattern Formation by Dewetting of Polymer Thin Film. Prog. Polym. Sci. 2011, 36, 269-293.

(46) Brassat, K.; Lindner, J. K. N. Nanoscale Block Copolymer SelfAssembly and Microscale Polymer Film Dewetting: Progress in Understanding the Role of Interfacial Energies in the Formation of Hierarchical Nanostructures. Adv. Mater. Interfaces 2020, 7, 1901565.

(47) Maiti, A.; Gunreddy, V.; Schaumont, P. A Systematic Method to Evaluate and Compare the Performance of Physical Unclonable Functions. In Embedded Systems Design with FPGAs; Athanas, P., Pnevmatikatos, D., Sklavos, N., Eds.; Springer: New York, 2013; pp 245-267.

(48) Bassham, L.; Rukhin, A.; Soto, J.; Nechvatal, J.; Smid, M.; Barker, E.; Leigh, S.; Levenson, M.; Vangel, M.; Banks, D.; Heckert, A.; Dray, J.; Vo, S. A Statistical Test Suite for Random and Pseudorandom Number Generators for Cryptographic Applications; National Institute of Standards and Technology (NIST), special publication, 2010; 800-22 Rev 1a.

(49) Maes, R.; van der Leest, V.; van der Sluis, E.; Willems, F. Secure Key Generation from Biased PUFs. In Cryptographic Hardware and Embedded Systems-CHES 2015; Güneysu, T., Handschuh, H., Eds.; Lecture Notes in Computer Science; Springer: Berlin, Heidelberg, 2015; Vol. 9293.

(50) Kwok, S. H.; Ee, Y. L.; Chew, G.; Zheng, K.; Khoo, K.; Tan, C. H. A Comparison of Post-Processing Techniques for Biased Random Number Generators. In Information Security Theory and Practice. Security and Privacy of Mobile Devices in Wireless Communication. WISTP 2011; Ardagna, C. A., Zhou, J., Eds.; Lecture Notes in Computer Science; Springer: Berlin, Heidelberg, 2011; Vol. 6633.

(51) von Neumann, J. Various Techniques Used in Connection with Random Digits. In Applied Math Series 12; National Bureau of Standards: USA, 1951. 
(52) Sakir, M.; Pekdemir, S.; Karatay, A.; Küçüköz, B.; Ipekci, H. H.; Elmali, A.; Demirel, G.; Onses, M. S. Fabrication of Plasmonically Active Substrates Using Engineered Silver Nanostructures for SERS Applications. ACS Appl. Mater. Interfaces 2017, 9, 39795-39803.

(53) Kiremitler, N. B.; Pekdemir, S.; Patarroyo, J.; Karabel, S.; Torun, I.; Puntes, V. F.; Onses, M. S. Assembly of Plasmonic Nanoparticles on Nanopatterns of Polymer Brushes Fabricated by Electrospin Nanolithography. ACS Macro Lett. 2017, 6, 603-608.

(54) Ting, Y.-H.; Park, S.-M.; Liu, C.-C.; Liu, X.; Himpsel, F. J.; Nealey, P. F.; Wendt, A. E. Plasma Etch Removal of Poly(methyl methacrylate) in Block Copolymer Lithography. J. Vac. Sci. Technol, B: Microelectron. Nanometer Struct.-Process., Meas., Phenom. 2008, 26, 1684-1689.

(55) Ferrarese Lupi, F.; Giammaria, T. J.; Miti, A.; Zuccheri, G.; Carignano, S.; Sparnacci, K.; Seguini, G.; De Leo, N.; Boarino, L.; Perego, M.; Laus, M. Hierarchical Order in Dewetted Block Copolymer Thin Films on Chemically Patterned Surfaces. ACS Nano 2018, 12, 7076-7085.

(56) Hur, S.-M.; Onses, M. S.; Ramírez-Hernández, A.; Nealey, P. F.; Rogers, J. A.; de Pablo, J. J. Interplay of Surface Energy and Bulk Thermodynamic Forces in Ordered Block Copolymer Droplets. Macromolecules 2015, 48, 4717-4723.

(57) Castle, S. R.; Dalay, T. S.; Muyskens, R. H.; Navarrete, N. G.; Nystrom, B. D.; Pangburn, T. E.; Rhodus, S. L. Device and Method for Scanning the Physical Signature Data of a Physical Unclonable Function with a Smartphone. U.S. Patent 20,200,304,321 A1, 2020.

(58) Cheng, H.; Lu, Y.; Zhu, D.; Rosa, L.; Han, F.; Ma, M.; Su, W.; Francis, P. S.; Zheng, Y. Plasmonic Nanopapers: Flexible, Stable and Sensitive Multiplex PUF Tags for Unclonable Anti-Counterfeiting Applications. Nanoscale 2020, 12, 9471-9480.

(59) Han, F.; Liu, Y.; Li, F.; Lu, Y.; Cheng, H.; Lin, Y.; Zhao, T.; Ng, S. H.; Bach, U.; Zheng, Y. Self-Assembly of Coordination Polymers on Plasmonic Surfaces for Computer Vision Decodable, Unclonable and Colorful Security Labels. J. Mater. Chem. C 2019, 7, 13040-13046. 